\title{
The MADS transcription factor GhAP1 coordinates the flowering regulatory pathway in upland cotton (Gossypium hirsutum L.)
}

\section{Xiaoqian Cheng}

Chinese Academy of Agricultural Sciences Cotton Research Institute

Hantao Wang

Cotton Research Institute

Hengling Wei

Cotton Research Institute

Lijiao Gu

Cotton Research Institute

Pengbo Hao

Cotton Research Institute

Huiru Sun

Cotton Research Institute

Aimin Wu

Cotton Research Institute

Shuaishuai Cheng

Cotton Research Institute

Jinping Hua

chinese agricultural university

Shuxun Yu (D ysx195311@163.com)

Chinese Academy of Agricultural Sciences Cotton Research Institute https://orcid.org/0000-00029715-3462

\section{Research article}

Keywords: GhAP1, Arabidopsis, VIGS, Yeast, Cotton, Early flowering

Posted Date: December 3rd, 2019

DOI: https://doi.org/10.21203/rs.2.18061/v1

License: (c) (1) This work is licensed under a Creative Commons Attribution 4.0 International License.

Read Full License 


\section{Abstract}

Background: MADS-box gene family plays an important role in the molecular regulatory network of flower development. APETAL1 (AP1), a MADS-box gene, plays an important role in the development of flower organs. Although many studies about MADS-box family genes have been reported, the function of AP1 is still not clear in cotton. Results: In this study, GhAP1 (Gh_D03G0922), a candidate gene for cotton flower time and plant height obtained from our previous studies, was cloned from CCRI50 cotton variety and functionally characterized. Subcellular localization demonstrated that GhAP1 was located in nucleus. Infection test of Arabidopsis revealed that GhAP1 could cause precocious flowering and virus-induced gene silence (VIGS) assay demonstrated that GhAP1 could lead to delayed flowering of cotton plants. Yeast one-hybrid assays and transient dual-luciferase assays suggested that floral meristem identity control gene LEAFY (LFY) can bind the promoter of GhAP1 and negatively regulate it. Yeast two-hybrid assays suggested that GhAP1 can interact with pyridoxal phosphate (PLP)-dependent transferases superfamily protein PSAT2. Conclusions: Our research indicated that GhAP1 might work as a positive regulator in plant flowering. Moreover, GhAP1 may interact with GhPSAT2 and be negatively regulated by GhLFY in the regulatory pathways. This work laid the foundation for subsequent functional studies of GhAP1.

\section{Background}

Flowering is one of the important traits in plants. In 1990, Yanofsky et al. cloned an AGAMOUS gene for floral organ development from Arabidopsis for the first time, then research on flower development has developed rapidly [1]. Usually, the flowers of typical dicotyledonous plants consist of sepal, petal, stamen, pistil and carpel. These five floral organs are controlled by organ characteristic genes. The flower growth $A B C$ model revealed the molecular mechanism underlying flower development which was accepted generally [2]. With the deepening of research, $D$ functional genes were found in Petunia hybrida and $E$ functional genes were found in Gerbera hybrida and Oryza sativa [3-5], then ABCDE model or AE model about flower was derived.

MADS-box genes are widely distributed in plants, and more than one hundred genes are found in Arabidopsis [6, 7]. Based on the structural differences, MADS-box genes can be divided into Type $\mathbb{Z}$ and

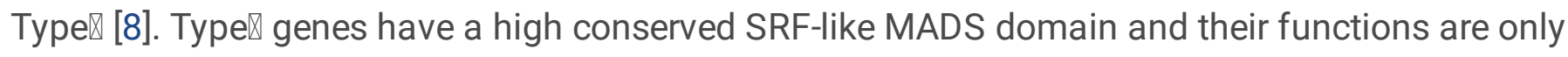

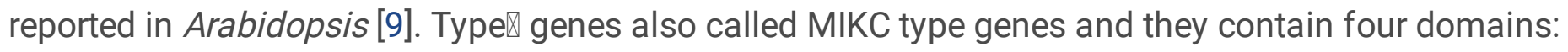
MEF2-like MADS, Intervening (I), Keratin (K), and C-terminal (C) domains. MADS domain is located in Nterminal consisting of 56 amino acids and is the most conservative domain [10]. I domain is less conservative, which is related with combination of DNA and protein [11]. According to different structures of I domain, these genes can be subdivided into $\operatorname{MIKC}^{C}$ and $\operatorname{MIKC}^{*}[12,13] . K$ domain is a semiconservative region, it is homologous to the helix of keratin and has about 70 amino acids [14] and is the signature sequence of MIKC type MADS-box genes. Expect for individual genes of maize, all the MADSbox genes contain $\mathrm{K}$ domain in plants [15]. The $\mathrm{C}$ domain is the least conservative region comes after the $\mathrm{K}$ domain, which is a transcriptional activation region composed of hydrophobic amino acids [16]. 
Different $\mathrm{C}$ domains can lead to different functions [17]. The MADS-box gene family encodes transcription factors (TFs) that shares a highly conserved domain which can bind CArG box in promoter region of the MADS-box protein target genes [18].

MADS-box genes play important regulatory roles in plant growth and development. Most of them play important roles in the development of plant reproductive organs and some of them participate in the development of plant vegetative organs [19]. In different species, MADS-box genes have different degree of sub-functionalization but most of them are related to flowering. As a member of the MADS-box gene family, the function of $A P 1$ in plants is very conservative. FUL (AGL8) promotes the initiation of flower development with SUPPRESSOR OF OVEREXPRESSION OF CONSTANS 1 (SOC1) in the regulation of flowering time in Arabidopsis [20]. In the photoperiod dependent flowering pathway, FUL is involved in regulating the transformation of bud meristems into inflorescence meristems, and then the expression of FUL and SOC1 in the developed inflorescence procambium induces the transformation to flower meristems, thus controlling the flowering time [21]. Recent studies showed that PasAP1 is expressed during early development of tendrils with the A function. PasAP1 appears to play an important role in the tendril development and in maintaining the tissues with meristem characteristics [22]. NnAP1 shares 75$96 \%$ similarity with other plant $A P 1 s$ and it plays an important role in floral meristem and floral organ formation [23]. AP1 was identified as a floral meristem related gene that regulates flowering in many plant species, including Moso bamboo, Cymbidium ssp and Dendrobium nobile species [24-26]. However, few studies have been done in cotton.

Cotton is the one of most important economic crop that is widely cultivated throughout the world [27]. The MADS-box genes have been extensively studied in cotton. Sarah Muniz Nardeli et al. identified a total of 207 MADS-box genes in tetraploid cotton, which opens an avenue to understanding the origin and evolution of each gene subfamily within diploid and polyploid species and paves the way for functional studies in cotton species [28]. The MIKC-type genes of MADS-box family in upland cotton were identified by Zhongying Ren et al. [9]. Additionally, 110 GhMIKC genes were identified and phylogenetically classified into 13 subfamilies, however, the Flowering locus C (FLC) subfamily was absent in the upland cotton [9]. It was found that GhmiR157/SPL promoted the development process of flower organs by regulating MADS-box genes [29]. Overexpression of both GhSOC1 and GhMADS42 in Arabidopsis accelerated flowering, and GhSOC1 can interact with AP1 protein in cotton [30]. Besides, GhMADS3 [31], GhMADS9 [32], GhMADS11 [33] have been studied in cotton.

Early maturity is one of the important traits of cotton. Early-maturing cotton is helpful for realizing both grain and cotton maturity, reducing the land conflict between grain and cotton. Flowering is one of the most important traits of early maturity. Many studies have shown that MADS TFs play important roles in all the stages of plant growth, especially flowering. Therefore, it is important to study the functional and molecular mechanism of MADS genes in cotton. In our previous studies of our lab, a MIKC type MADS gene, GhAP1 was identified as a candidate gene for cotton flower time [34] and plant height [35]. In this study, GhAP1 was cloned and characterized. The overexpression lines of GhAP1 in Arabidopsis and the VIGS plants of GhAP1 in cotton were used to explore its biological function. Yeast one-hybrid assays, 
transient dual-luciferase assays and yeast two-hybrid assays were performed to examine the molecular mechanism of GhAP1. Our findings help to enrich flowering regulatory pathways and provide genetic resources for genetic improvement of cotton varieties.

\section{Results}

\section{Gene structure and protein sequence analysis of GhAP1}

Based on the results of genome-wide association study (GWAS) in our previous studies, GhAP1 (Gh_D03G0922) was selected according to gene annotation, and its Arabidopsis homologs contains MADS-box domains [35]. GhAP1 was located on D03 chromosome and its Arabidopsis homolog gene is Agamous-like MADS-box protein AGL8 (AT5G60910). The protein sequence similarity between GhAP1 and AGL8 was $56.2 \%$. The genome DNA length was $5452 \mathrm{bp}$ and the CDS length was $690 \mathrm{bp}$, encoding 229 amino acids (Fig. 1a). In addition, GhAP1 contained eight exons and seven introns (Fig. 1a). Protein sequence analysis showed its homologous genes of different species shared the high conserved MADS domain and the signature K-domain of MIKC type MADS-box genes. A putative nuclear localization signal (NLS) sequence (ALKRIRSRKNQLM) was predicted in GhAP1 protein sequence (Fig. 1b).

\section{Expression analysis of GhAP1 in different tissues and different varieties}

Quantitative real-time PCR (qRT-PCR) was performed to detect the expression levels of GhAP1 in roots, stems, leaves, buds, fiber, petals, and sepals. GhAP1 was found to be differentially expressed in different tissues. GhAP1 was strongly expressed in sepals and leaves but was weakly expressed in petals, roots, and fiber (Fig. 2a). To evaluate the expression pattern of GhAP1 during different growth stages, the expression level of GhAP1 was examined in the leaves of two early maturing varieties (CCRI50 and CCRI74) and two late maturing varieties (BM and G11) at different growth stages. The results revealed that the expression level of GhAP1 reached its maximum level in CCRI50 plants at the three-leaf stage. In addition, the expression levels of GhAP1 increased gradually and then decrease during growth stages and were higher in early maturing varieties than in late maturing varieties (Fig. 2b).

\section{GhAP1 was localized to the nucleus}

GhAP1 was predicted by subcellular location software Plant-mPloc (http://www.csbio.sjtu.edu.cn/bioinf/plant-multi/) and the results showed that the GhAP1 protein was localized to the nucleus. To confirm our prediction, the 35S-GhAP1::GFP vector was constructed and injected into tobacco leaves. Using a fluorescence microscope, we found that the tobacco leaves harboring the 35S-GhAP1::GFP construct emitted green fluorescence predominantly in nuclei under blue light (Fig. 3a, 3b).

\section{Overexpression of GhAP1 promoted flowering in Arabidopsis}

To further study the function of GhAP1, the overexpression vector 35S::GhAP1 was constructed and transformed into Arabidopsis. qRT-PCR results showed that GhAP1 gene was hardly expressed in WT, but 
was highly expressed in transgenic plants, which was significantly higher in transgenic plants than that of non-transgenic Arabidopsis plants (Fig. 4a). Early flowering was observed in GhAP1-OE transgenic Arabidopsis plants (Fig. 4b). The flowering time was measured using the days after sowing (DAS) to first flowering. Through investigation, we found that WT plants started flowering at 29 DAS and had 11 rosette leaves, whereas transgenic lines started flowering at 24 DAS and had 8 rosettes. The transgenic Arabidopsis bloomed about 5 days earlier than the WT and had 3 rosette leaves less than WT (Table 1).

Table 1. Flowering time and rosette leaf number of transgenic and WT Arabidopsis lines

\begin{tabular}{llll} 
Plant lines & Start bolting(DAS) & Start flowering(DAS) & Number of rosette leaves \\
\hline WT & $27.3 \pm 0.90$ & $29.6 \pm 1.20$ & $11.6 \pm 1.11$ \\
\hline GhAP1-4 & $20.6 \pm 0.49$ & $25.6 \pm 0.80$ & $8.4 \pm 0.49$ \\
\hline GhAP1-15 & $19.7 \pm 0.90$ & $24.7 \pm 0.46$ & $8.3 \pm 0.90$ \\
\hline GhAP1-17 & $19.4 \pm 1.36$ & $24.6 \pm 0.49$ & $9 \pm 0.63$
\end{tabular}

* Data were shown as means \pm SDs from ten plants.

\section{VIGS delayed cotton flowering}

SGN-VIGS Tool (http://vigs.solgenomics.net/) was used to analyze the GhAP1 sequence and $250 \mathrm{bp}$ of GhAP1 was selected to construct PCLCrVA-GhAP1 vector. PCLCrVA-PDS vector was served as a positive control and pCLCrVA vector was served as an empty control. The cotton plants harboring pCLCrVA-PDS showed an albino phenotype, suggesting that the VIGS assay was successful. qRT-PCR was performed to evaluate the effect of gene silencing, the results showed that the expression level of GhAP1 in positive plants was significantly lower than that in VA empty control (Fig. 5a). When flowering was observed in VA plants, the positive plants were not (Fig. 5b). Compared to VA plants, we found that positive plants flowered later.

\section{GhAP1 interacted with GhPSAT2}

A yeast library was constructed using the bud samples from two-leaf, three-leaf and five-leaf stages of early maturing variety CCRI50. pGBKT7-GhAP1 vector was build and transformed into Y2H Gold yeast cells. After three days both of pGBKT7 control and pGBKT7-GhAP1 can grow on SD-Trp medium plate, but can't grow and become blue on SD-Trp/-His/-Ade and SD-Trp/-His/-Ade/X-a-gal medium plate (Fig. $6 a)$, indicating that GhAP1 had no self-activation activity. According to gene annotation and yeast twohybrid validation, a pyridoxal phosphate (PLP)-dependent transferases superfamily protein GhPSAT2 (Gh_D07G1721) was identified to be interacted with GhAP1 (Fig. 6b). The research on GhPSAT2 was poor, but its homologous gene in soybean was proved to encode mitochondrial isozyme of aspartate aminotransferase (AAT4) when expressed in E. coli [36]. 
Three GhLFY binding sites (CCAATGG) were founded in promoter of GhAP1, which located at $-80,-1500$ and $-1700 \mathrm{bp}$. In order to validate which site can interact with GhLFY, approximately $51 \mathrm{bp}$ around these sites were selected respectively and inserted into the pHis2 carrier vector with three series repeats (called pHis2-L plasmid). When 120 mM 3-amino-1,2,4-triazole (3-AT) were added, their self-activation could be inhibited. pGADT7-GhLFY vector was constructed and co-transformed into Y187 yeast cells with pHis2-L vector. The yeast cells contained pHis2-1500bp and pGADT7-GhLFY vector could growth on the SD-Trp/$\mathrm{His} /-\mathrm{Leu} / 120 \mathrm{mM} 3-\mathrm{AT}$ medium. These results indicate that GhLFY can directly bind the promoter of GhAP1 at the -1500 bp site (Fig. 7).

\section{The expression of GhAP1 was suppressed by GhLFY in the dual-luciferase assay}

A transient dual-luciferase assay was performed in tobacco leaves to elucidate the functional role of GhAP1 in vivo. The experiment was conducted using a double reporter plasmid, pGreenlI0800-LUC, containing the REN luciferase driven by the $35 \mathrm{~S}$ promoter and the LUC luciferase driven by the GhAP1 promoter. In addition, the assay includes an effecter plasmid, pGreenll62-SK, expressing the GhLFY. The constructs are shown in Fig. 8a. The ratio of LUC to REN was used to reflect the transcriptional activity. The results showed that compared with the control, GhLFY inhibited the GhAP1 promoter by significantly reducing the LUC/REN ratio (Fig. 8b), suggesting that GhLFY inhibited the expression of GhAP1.

\section{Discussion}

\section{Functional characterization of GhAP1 gene in cotton}

As an important commercial crop, cotton plays an important role in the development of the world's agriculture. Flowering is one of the important traits of cotton. A number of studies have shown that MADS TFs regulate numerous physiological and biochemical processes in plants [37-39]. It involves every stage of the plant's growth cycle and is considered to be a key gene family regulating flowering and vegetative organ development [40]. In this study, a MADS-box gene GhAP1 was isolated and characterized from upland cotton CCRI50. Sequence analysis revealed that GhAP1 contained a high conserved MADS domain and a moderate conserved K-box. These typical domains suggested that GhAP1 was a MICK type gene. The MIKC family members are plant specific TFs, and play crucial roles in plant seed development and floral identification [9]. Organizational expression patterns were analyzed by qRT-PCR. The results showed that GhAP1 was highly expressed in sepals, which was the same as described in the classic ABCDE model: the formation of sepals was determined by the formation of $A P 1 / A P 1$ homologous dimer and SEP/SEP dimer [41]. The GhAP1 gene had highest expression at the three-leaf stage, and had higher expression in early maturing cultivars than in the late-maturing cultivars. Our GhAP1 gene shared $72.27 \%$ similarity with the gene reported by Su et al. [34]. In addition, consistent with the conclusions of Su et al., GhAP1 presented similar expression pattern to Su et al. gene [34]. Subcellular localization analysis revealed that the GhAP1 protein was located in nucleus, consistent with the website predicted. 
As a class of $A$ functional gene, $A P 1$ plays an important role in the normal formation of flower organs. We carried out Arabidopsis transformation and cotton VIGS experiments. The overexpression showed that GhAP1 could promote the flowering of Arabidopsis, and qRT-PCR indicated that the expression of GhAP1 in transgenic plants was much higher than that in WT plants. OSMADS18 was a homologous gene of GhAP1 in rice, and overexpression of OSMADS18 in rice can induce early flowering [42]. Overexpression of $P a A P 1$ in Arabidopsis can trigger early flowering in the 35S::PaAP1 transgenic Arabidopsis lines [43]. GMAP1 could cause early flowering and alteration of floral organs when it was ectopically expressed in tobacco [44]. These studies are similar to the results of this study. In the VIGS assay, compared with the control group, the experimental group showed a late flower phenotype, and the expression level of GhAP1 in the experimental group was significantly lower than that of the VA plants. Silencing the SBP-box gene in Snapdragon by VIGS technology will down-regulate the expression of multiple MADS-box genes, such as SQUA, AmFUL, etc., resulting in late flowering or even no flowering of plants [45]. Silencing of TaAG-A and $T a A G B$ in a fertile wheat line resulted in green and yellow striped leaves, emaciated spikes and reduced seed setting rate [46]. These studies increased the credibility of our study.

\section{Molecular mechanism of GhAP1}

Proteins interact with each other to diversify their functions. Yeast hybrid system is an efficient and rapid method for analyzing protein interactions. For example, OsMADS18 can interact with the seed-specific protein NF-YB by yeast two-hybrid system [47]. In addition, MADS-box proteins can regulate the temporal and spatial expression of target genes, and its own expression is also strictly regulated.

Previous reports showed that $A P 1$ can be directly and positively regulated by LFY [48]. Promoter sequence analysis revealed that three GhLFY-binding sites (CCAATGG) were present in the promoter region of GhAP1. Yeast one-hybrid assay showed that GhLFY can interacte with the -1500 bp promoter site of GhAP1, indicating that GhLFY can regulate GhAP1 by binding to the -1500 bp site. By dualluciferase assay, it was found that the value of LUC/REN in the experimental group was significantly lower than that of the control group, indicating that GhLFY might negatively regulate GhAP1 expression. This is in contrast to the reporting pathway in Arabidopsis [49]. This may be due to the functional redundancy of GhLFY and GhAP1, which is considered to be implemented by a set of shared genes. Moreover, studies have shown that GhLFY and GhAP1 have antagonistic effects on target gene regulation [48]. Therefore, we suspected that this is an antagonist effect that leads to this result. As a complex biological phenomenon of plants, flowering was regulated by numerous genes. Therefore, it's understandable that there are different results, but this conjecture remains to be further proved.

As a TF, AP1 can regulate the expression of target genes, but most studies on signaling pathways comes to an abrupt end with AP1. However, little research has been done on its mechanism of action in cotton. In our study, the library screening experiment was carried out by using yeast two-hybrid. We found that a protein GhPSAT2 can interact with GhAP1. It is a mitochondrial isozyme of aspartate aminotransferase AAT4 in soybean [36]. Currently, there are few reports on GhPSAT2, indicating that it may be a new gene, and its mechanism of action on plant growth still needs to be further studied. 


\section{Conclusions}

GhAP1 is a TF associated with flowering. It has a highly conserved MADS domain and belongs to the MADS-box gene family. GhAP1 is located in the nucleus. Overexpressed GhAP1 can promote flowering in Arabidopsis. The cotton VIGS showed a delayed flowering phenotype. Yeast two-hybrid assay showed that GhAP1 can interact with GhPSAT2. GhAP1 promoter contains GhLFY binding sites and GhLFY can interact with the promoter of GhAP1. In addition, GhAP1 is negatively regulated by GhLFY.

\section{Methods}

\section{Plant materials and growth conditions}

All plants used in our experiments were grown in a greenhouse at $25^{\circ} \mathrm{C}$, with a $16 \mathrm{~h}$ light/ $8 \mathrm{~h}$ dark cycle.

To examine gene expression at different stages of different maturity varieties, the leaves were collected from CCRI50, CCRI74, G11 and BM at the cotyledon and 1-, 2-, 3-, 4- and 5-leaf stages. Each sample included three repetitions. The growth period of CCRI50 is about 105 days. After Shuxun Yu and others investigation found that it has an obvious character of early maturity [50]. The growth period of CCRI74 is 100 days and it has obvious early maturity [51]. The growth period of G11 is about 123 days, which was identified by Guoxin rural technical service association [52]. BM plants have the characteristics of late emergence, tall and loose, it belongs to late maturing varieties [53]. As Gu described, these varieties are often used for testing and preservation in our laboratory [54]. All samples were quickly frozen in liquid nitrogen and stored at $-80^{\circ} \mathrm{C}$ for subsequent experiments. In addition, two early-maturing varieties, CCRI50 and CCRI74, and two non-early-maturing varieties, G11 and BM, were used for expression pattern analysis. The cotton varieties were planted in the field of the Cotton Research Institute of the Chinese Academy of Agricultural Sciences (Anyang, Henan, China). Different tissues, including roots, stems, leaves, buds, fiber, petals and sepals were harvested from CCRI50 [54].

\section{Gene cloning and sequence analysis}

To amplify the CDS and promoter of GhAP1 (Gh_D03G0922), we designed primers using Oligo7. The primers used in this study are listed in Additional file 1: Table S1. The full-length CDS and promoter fragment of GhAP1 was cloned from CDNA and DNA of CCRI50 leaves, respectively. The fragments were inserted into PBI121 vector and transformed into Escherichia coli competent cells (E. coli DH5a) for sequencing. The genomic and coding sequences of GhAP1 were submitted to Gene Structure Display Server online software (GSDS2.0) (http://gsds.cbi.pku.edu.cn/) to predict gene structures. Multiple sequence alignment was conducted using DNAMAN software.

\section{qRT-PCR}

Total RNA was isolated using RNA prep Pure Plant Kit (Polysaccharides \& Polyphenolics-rich) (Tiangen, China). One microgram of total RNA was prepared for cDNA synthesis in a $20 \mu \mathrm{l}$ reaction system using a 
Prime Script ${ }^{\text {TM }}$ RT reagent kit with gDNA Eraser (RR047A) (TaKaRa, Japan). The cDNA was diluted 5 times for qRT-PCR. Transcript levels were detected using a 7500 Real-Time PCR system (Applied Biosystems) and UltraSYBR Mixture (Low ROX) (CW2601M) (CWBIO, China). The $10 \mu$ reaction volume contained the following components: $5 \mu \mathrm{l}$ of $2 \times U$ traSYBR Mixture, $0.2 \mu$ of the PCR forward primer $(10 \mu \mathrm{M}), 0.2 \mu \mathrm{l}$ of the PCR reverse primer $(10 \mu \mathrm{M}), 1 \mu \mathrm{l}$ of $\mathrm{cDNA}$ and $3.6 \mu \mathrm{l}$ of $\mathrm{ddH}_{2} \mathrm{O}$. The optimal PCR amplification procedure was used as follows: a pre-denaturation step at $95^{\circ} \mathrm{C}$ for $10 \mathrm{~min} ; 40$ cycles of $95^{\circ} \mathrm{C}$ for $10 \mathrm{~s}$, $60^{\circ} \mathrm{C}$ for $30 \mathrm{~s}$ and $72^{\circ} \mathrm{C}$ for $32 \mathrm{~s}$, a melting curve step at $95^{\circ} \mathrm{C}$ for $15 \mathrm{~s}, 60^{\circ} \mathrm{C}$ for $1 \mathrm{~min}, 95^{\circ} \mathrm{C}$ for $15 \mathrm{~s}$ and $60^{\circ} \mathrm{C}$ for $15 \mathrm{~s}$. GhActin and $A t U B Q 10$ were used as reference genes. The $2^{-\triangle \triangle C T}$ method was applied to calculate relative expression levels [55]. All reactions were performed with three technical replicates.

\section{Subcellular localization}

The CDS sequence of GhAP1 without termination code was amplified from CCRI50 cDNA using PCR. GhAP1 was cloned into PBI121-GFP to construct 35-GhAP1::GFP vector and transformed into Agrobacterium tumefaciens strain LBA4404. Approximately $200 \mu \mathrm{LBA4404}$ strains were added into LB liquid medium containing kanamycin, rifampicin and streptomycin, shaking to $O D 600=1.8-2.0$ at $28^{\circ} \mathrm{C}$. The culture was centrifuged for $10 \mathrm{~min}$ at $4000 \mathrm{rpm}$. Adjust the OD600=1.5 using the transformation medium. The bacteria were left in the dark at room temperature for $3 \mathrm{~h}$ before injected into the tobacco leaves. The fluorescent signal was observed by fluorescence microscope after 2 days (dark culture for 24 $\mathrm{h}$ and normal culture for $24 \mathrm{~h}$ ).

\section{Genetic transformation of Arabidopsis}

The CDS sequence of GhAP1 was inserted into PBI121 vector to construct 35S::GhAP1 and transformed into the Agrobacterium tumefaciens strain LBA4404 chemically competent cells. Arabidopsis was infected by dipping flower method [56]. The LBA4404 cells containing 35S::-GhAP1 were cultured in the liquid medium and shaken to $O D 600=1.2-1.6$ at $28^{\circ} \mathrm{C}$. The culture was centrifuged for $5 \mathrm{~min}$ at $5000 \mathrm{rpm}$ to remove the supernatant. The bacteria was resuspended using a transformation medium $(0.217 \mathrm{~g}$ $1 / 2 \mathrm{MS}+5 \mathrm{~g}$ sucrose $+20 \mu \mathrm{l}$ silwettl-77 per $100 \mathrm{ml} \mathrm{H}_{2} \mathrm{O}$ ) to adjust the OD600 $=0.8-1.0$. The flower buds of WT plants were immersed in the suspension for $50 \mathrm{~s}$ and the transformed plants were placed in the dark condition for $24 \mathrm{~h}$. After one week, the Arabidopsis plants were genetically transformed again. The seeds harvested form the transformed WT plants are T0 generation. The positive plants were screened using the 1/2MS medium containing kanamycin. Phenotypic observation and data statistics were carried out in $\mathrm{T}_{3}$ generation.

\section{VIGS assay}

For the VIGS assay, approximately 250 bp fragment of GhAP1 gene was amplified from the CCRI50 cDNA and integrated into the pCLCrVA vector to construct pCLCrVA-GhAP1. The pCLCrVA-GhAP1 plasmids were transformed into LBA4404. The LBA4404 strains carrying pCLCrVA-GhAP1, pCLCrVA (negative control) or pCLCrVA-PDS (positive control) were mixed with the strain harboring pCLCrVB (helper vector) (1:1 ratio, OD600 $=1.5$ ) and co-injected into two fully expanded cotyledons of TM-1 plants. In the VIGS assay, at 
least 20 seedlings were used per group. For qRT-PCR detection, samples from at least 6 uniform injected plants were used. The cotton plants were cultivated in a greenhouse at $22^{\circ} \mathrm{C}$ with a $16 \mathrm{~h}$ light/ $8 \mathrm{~h}$ dark cycle. The experiment was repeated three times. The detailed VIGS procedure was performed as previously described $[57,58]$.

\section{Yeast two-hybrid}

To identify the interaction proteins of GhAP1, the buds and leaves of CCRI50 from the two-leaf, three-leaf and five-leaf stages were used to construct yeast two-hybrid library. GhAP1 was cloned into pGBKT7 to construct pGBKT7-GhAP1 plasmids and transformed into $\mathrm{Y} 2 \mathrm{H}$ yeast receptor cells. Three colonies were randomly selected for self-activation detection. The pGBKT7 empty vector was used as a negative control. The three clones were diluted with water and placed on the defect medium of yeast growth, namely, SD/-Trp, SD/-Trp/-His/-Ade and SD/-Trp/-His/-Ade/X-a-gal.

The mating method was used to screen yeast library on SD/-Trp/-His/-Ade/-Leu plates to identify the proteins that can interact with GhAP1. The $\mathrm{Y} 2 \mathrm{H}$ yeast transformants containing the correct pGBKT7GhAP1 was used to prepare the yeast competent cells, and the library plasmid pGBKT7-cDNA was transferred into it. Screening was conducted on the defective culture plate of SD/-Trp/-His/-Ade/-Leu plates, and the normally growing spots were coated on the culture plate of SD/-Trp/-His/-Ade/-Leu with Xa-Gal for further screening. The spots that could grow normally and turn blue were considered to be positive spots. The positive clones were amplified via PCR and sequenced. Cotton genome blast analysis was performed to obtain the coding genes of potential proteins [59].

\section{Yeast one-hybrid assay}

A suitable 50 bp promoter fragment sequence of GhAP1 was selected and three copies were designed to construct pHis2 recombinant vector. The recombinant plasmid was called pHis2-L. pHis2-L construct, positive control and negative control were applied to the corresponding defect culture plates ( $p$ GAD53m+pHIS2 as negative control, pGAD53m+p53HIS as positive control). Different concentrations of 3-AT were added to the defect culture plate to screen the appropriate 3-AT concentration. The CDS sequence of GhLFY was inserted into the pGADT7 vector to construct pGADT7-GhLFY and cotransformed into $Y 187$ yeast cells with pHis2-L constructs. The $Y 187$ yeast cells containing pGADT7GhLFY and pHis2-L were identified on SD/-Trp/-His/-Leu + 3-AT plates. If it can grow normally, it indicated that the GhLFY gene is the upstream gene of GhAP1.

\section{Dual-luciferase reporter assay}

Transient reporter expression was performed in tobacco leaves using a dual-luciferase reporter system [60]. The GhAP1 promoter was amplified and inserted into the pGreenII0800-LUC vector as the reporter plasmids. The ORF of GhLFY was amplified and inserted into the pGreenll62-SK vector as the effecter plasmids. The fusion construct plasmids were transformed into Agrobacterium tumefaciens strain GV3101 (pSoup-p19). The GV3101 (pSoup-p19) cells containing the recombinant plasmids were 
incubated in LB liquid medium containing $50 \mathrm{mg} \mathrm{L}^{-1}$ kanamycin, gentamycin and rifampin until the OD600 value reached $0.5-0.6$. Subsequently, the culture was adjusted to an OD600 value of 0.2 with the filtration buffer (10 mM MgCl $2,10 \mathrm{mM} \mathrm{MES}$ and100 $\mu \mathrm{M}$ acetosyringone). The culture suspensions were left for $2 \mathrm{~h}$ at room temperature. The effecter and reporter suspensions were mixed in a 1:1 ratio and coinfiltrated into tobacco leaves. After 2 days of infiltration, LUC and REN luciferase activity was detected using a dual-luciferase ${ }^{\circledR}$ reporter assay system (Promega, USA) on a Glomax 20/20 Luminometer (Promega, USA) according to the manufacturer's instructions. At least six independent replicates were performed.

\section{Abbreviations}

TF: Transcription factor; VIGS: Virus-induced gene silencing; CDS: Coding sequence; aa: amino acid; NLS: Nuclear localization signal; DAS: Days after sowing; WT: Wild type; qRT-PCR: Quantitative real-time PCR; Trp: Tryptophan; Leu: Leucine; Ade: Adenine; His: Histidine; 3-AT: 3-amino-1,2,4-triazole

\section{Declarations}

\section{Ethics approval and consent to participate}

The experimental research on plants (either cultivated or wild), including collection of plant material, complied with institutional, national, or international guidelines. Field studies were conducted in accordance with local legislation. The plant materials used in this study were previously preserved in our laboratory.

\section{Consent for publication}

Not applicable.

\section{Availability of data and materials}

The data sets supporting the results of this article are included within the article and its additional file.

\section{Competing interests}

The authors declare that they have no competing interests.

\section{Funding}

This research was support by the Shangdong TAISHAN Industry Leading Talent Program to Shuxun Yu (LJNY201608). The funders had no role in the design of the study, the collection, analysis, and interpretation of data, and in writing the manuscript.

\section{Authors' contributions}


S.X.Y. designed the research program. J.P.H., H.L.W., H.T.W. and L.J.G. revised the manuscript. X.Q.C. wrote the paper. X.Q.C., P.B.H. and A.M.W. performed the data analysis. X.Q.C., H.R.S. and S.S.C. performed the yeast two-hybrid test. X.Q.C. performed left experiments. All authors have read and approved the final manuscript.

\section{Acknowledgments}

We thank all authors for their contributions to the article. We also appreciate the reviewers and editors for their patience regarding this work.

\section{References}

1. Martin F. Yanofsky* HM, John L. Bowman, Gary N. Drews,, Meyerowitz* KAFEM: The protein encoded by the Arabidopsis homeotic gene agamous resembles transcription factors. nature 1990, 346:35-39.

2. M CESME: The war of the whorls: genetic interactions controlling flower development. Nature 1991, 353(6339):31-37.

3. Lucia Colombo aJF, a Emmo Koetje,a Jacques van Went,b Hans J. M. Dons,a, Gerco C. Angenent aaAJvT: The Petunia MADS Box Gene FBPI7 Determines Ovule Identity. The Plant Cell 1995, 7(11):1859-1868.

4. Angenent GC, Colombo L: Molecular control of ovule development. trends in plant science reviews 1996, 1(7):228-232.

5. H TGS: Floral quartets. nature 2001, 409:469-471.

6. De Bodt S, Raes J, Van de Peer Y, Theissen G: And then there were many: MADS goes genomic. Trends in plant science 2003, 8(10):475-483.

7. Parenicova L: Molecular and Phylogenetic Analyses of the Complete MADS-Box Transcription Factor Family in Arabidopsis: New Openings to the MADS World. The Plant Cell Online 2003, 15(7):15381551.

8. Becker A: The major clades of MADS-box genes and their role in the development and evolution of flowering plants. Molecular Phylogenetics and Evolution 2003, 29(3):464-489.

9. Ren Z, Yu D, Yang Z, Li C, Qanmber G, Li Y, Li J, Liu Z, Lu L, Wang L et al: Genome-Wide Identification of the MIKC-Type MADS-Box Gene Family in Gossypium hirsutum L. Unravels Their Roles in Flowering. Frontiers in plant science 2017, 8:384.

10. Immink RG, Gadella TW, Jr., Ferrario S, Busscher M, Angenent GC: Analysis of MADS box proteinprotein interactions in living plant cells. Proceedings of the National Academy of Sciences of the United States of America 2002, 99(4):2416-2421.

11. José Luis Riechmann, Wang M, Meyerowitz* EM: DNA-binding properties of Arabidopsis MADS domain homeotic proteins APETALA1, APETALA3, PISTILLATA and AGAMOUS. Nucleic Acids Research 1996, 24(16):3134-3141. 
12. Gunter Theißen, Jan T. Kim, Saedler H: Classification and Phylogeny of the MADS-Box Multigene Family Suggest Defined Roles of MADS-Box Gene Subfamilies in the Morphological Evolution of Eukaryotes. 1996, 43:484-516.

13. Kaufmann K, Melzer R, Theissen G: MIKC-type MADS-domain proteins: structural modularity, protein interactions and network evolution in land plants. Gene 2005, 347(2):183-198.

14. Hong Ma, Martin F. Yanofsky, Meyerowitz EM: AGL1-AGL6, an Arabidopsis gene family with similarity to floral homeotic and transcription factor genes. GENES \& DEVELOPMENT 1991, 5:484-495.

15. Achim Fischer NB, Heinz Saedler and Gunter TheiBlen: Chromosomal mapping of the MADS-box multigene family in Zea mays reveals dispersed distribution of allelic genes as well as transposed copies. Nucleic Acids Research, 1995, 23(11):1901-1911.

16. Goto THK: Complexes of MADS-box proteins are sufficient to convert leaves into floral organs. nature 2001, 409(6819):525-529.

17. Lamb RS, Irish VF: Functional divergence within the APETALA3/PISTILLATA floral homeotic gene lineages. Proceedings of the National Academy of Sciences of the United States of America 2003, 100(11):6558-6563.

18. JosiE Luis RIECHMANN BAK, AND ELLIOT M. MEYEROWITZ*: Dimerization specificity of Arabidopsis MADS domain homeotic proteins APETALA 1区APETALA 3区PISTILIATA and AGAMOUS. Proc Nat/ Acad Sci USA 1996, 93:4793-4798.

19. huang fang cy, yu deyue: research advances of MADS-box genes in plants. journal of Nanjing Agricultural University 2012, 35(5):9-18.

20. Rony Borner GK, John Chandler, Roland Gleißner, Ellen Wisman, Klaus Apel and, Melzer S: A MADS domain gene involved in the transition to flowering in Arabidopsis. The Plant Journal 2000, 24(5):591-599.

21. Melzer S, Lens F, Gennen J, Vanneste S, Rohde A, Beeckman T: Flowering-time genes modulate meristem determinacy and growth form in Arabidopsis thaliana. Nature genetics 2008, 40(12):14891492.

22. Hernandes-Lopes J, Sousa-Baena MS, Lemos RCC, Correa TCS, Van Sluys MA, Melo-de-Pinna GFA: Toward understanding inflorescence development and architecture in Passiflora: insights from comparative anatomy and expression of APETALA1. American journal of botany 2019.

23. Kong DZ, Shen XY, Guo B, Dong JX, Li YH, Liu YP: Cloning and expression of an APETALA1-like gene from Nelumbo nucifera. Genetics and Molecular Research 2015, 14(2):6819-6829.

24. Chang YY, Chiu YF, Wu JW, Yang CH: Four orchid (Oncidium Gower Ramsey) AP1/AGL9-like MADS box genes show novel expression patterns and cause different effects on floral transition and formation in Arabidopsis thaliana. Plant \& cell physiology 2009, 50(8):1425-1438.

25. Chen Z, Han Y, Ning K, Ding Y, Zhao W, Yan S, Luo C, Jiang X, Ge D, Liu R et al: Inflorescence Development and the Role of LsFT in Regulating Bolting in Lettuce (Lactuca sativa L.). Frontiers in plant science 2017, 8:2248. 
26. Zhang X, Wang Q, Yang S, Lin S, Bao M, Bendahmane M, Wu Q, Wang C, Fu X: Identification and Characterization of the MADS-Box Genes and Their Contribution to Flower Organ in Carnation (Dianthus caryophyllus L.). Genes 2018, 9(4).

27. Gu L, Wei H, Wang H, Su J, Yu S: Characterization and functional analysis of GhWRKY42, a group Ild WRKY gene, in upland cotton (Gossypium hirsutum L.). BMC genetics 2018, 19(1):48.

28. Nardeli SM, Artico S, Aoyagi GM, de Moura SM, da Franca Silva T, Grossi-de-Sa MF, Romanel E, AlvesFerreira M: Genome-wide analysis of the MADS-box gene family in polyploid cotton (Gossypium hirsutum) and in its diploid parental species (Gossypium arboreum and Gossypium raimondii). Plant physiology and biochemistry : PPB 2018, 127:169-184.

29. Wang L, Liu N, Wang T, Li J, Wen T, Yang X, Lindsey K, Zhang X: The GhmiR157a-GhSPL10 regulatory module controls initial cellular dedifferentiation and callus proliferation in cotton by modulating ethylene-mediated flavonoid biosynthesis. Journal of experimental botany 2018, 69(5):1081-1093.

30. Zhang X, Wei J, Fan S, Song M, Pang C, Wei H, Wang C, Yu S: Functional characterization of GhSoc1 and GhMADS42 homologs from upland cotton (Gossypium hirsutum L.). Plant science : an international journal of experimental plant biology 2016, 242:178-186.

31. Guo Y, Zhu Q, Zheng S, Li M: Cloning of a MADS Box Gene (GhMADS3) from Cotton and Analysis of Its Homeotic Role in Transgenic Tobacco. Journal of Genetics and Genomics 2007, 34(6):527-535.

32. Shao S-Q, Li B-Y, Zhang Z-T, Zhou Y, Jiang J, Li X-B: Expression of a cotton MADS-box gene is regulated in anther development and in response to phytohormone signaling. Journal of Genetics and Genomics 2010, 37(12):805-816.

33. Y. L, Ning H, Zhang Z, Wu Y, Jiang J, Su S, Tian F, Li X: A cotton gene encoding novel MADS-box protein is preferentially expressed in fibers and functions in cell elongation. Acta biochimica et biophysica Sinica 2011, 43(8):607-617.

34. Su J, Pang C, Wei H, Li L, Liang B, Wang C, Song M, Wang H, Zhao S, Jia X et al: Identification of favorable SNP alleles and candidate genes for traits related to early maturity via GWAS in upland cotton. BMC genomics 2016, 17:687.

35. Su J, Li L, Zhang C, Wang C, Gu L, Wang H, Wei H, Liu Q, Huang L, Yu S: Genome-wide association study identified genetic variations and candidate genes for plant architecture component traits in Chinese upland cotton. TAG Theoretical and applied genetics Theoretische und angewandte Genetik 2018, 131(6):1299-1314.

36. Gregory J. Wadsworth 1 JSGaBFM: Characterization of a soybean cDNA clone encoding the mitochondrial isozyme of aspartate aminotransferase, AAT4. Plant Molecular Biology 1995, 27:10851095.

37. Colombo M, Masiero S, Vanzulli S, Lardelli P, Kater MM, Colombo L: AGL23, a type I MADS-box gene that controls female gametophyte and embryo development in Arabidopsis. The Plant journal : for cell and molecular biology 2008, 54(6):1037-1048.

38. Nobuhiro Kotoda a, Masato Wadaa, Shinnosuke Kusaba b, Yuriko Kano-, Murakamib TM, Junichi Soejima a: Overexpression of MdMADS5,an APETALA1-like gene of apple, causes early flowering in 
transgenic Arabidopsis. plant science 2002, 162:679-687.

39. Kang IH, Steffen JG, Portereiko MF, Lloyd A, Drews GN: The AGL62 MADS domain protein regulates cellularization during endosperm development in Arabidopsis. Plant Cel/ 2008, 20(3):635-647.

40. Zeng X, Liu H, Du H, Wang S, Yang W, Chi Y, Wang J, Huang F, Yu D: Soybean MADS-box gene GmAGL1 promotes flowering via the photoperiod pathway. BMC genomics 2018, 19(1):51.

41. Theissen LGaG: A hitchhiker's guide to the MADS world of plants. Gramzow and Theissen Genome Biology 11:214 2010, 11(6):214-225.

42. Fornara F, Pařenicová L, Falasca G, Pelucchi N, Masiero S, Ciannamea S, Lopez-Dee Z, Altamura MM, Colombo L, Kater MM: Functional Characterization of OSMADS18, a Member of the AP1/SQUA Subfamily of MADS Box Genes. Plant physiology 2004, 135(4):2207-2219.

43. Wang J, Zhang X, Yan G, Zhou Y, Zhang K: Over-expression of the PaAP1 gene from sweet cherry (Prunus avium L.) causes early flowering in Arabidopsis thaliana. J Plant Physiol 2013, 170(3):315320.

44. Yu YCFHHLSYD: An APETALA1-like gene of soybean regulates flowering time and specifies floralorgans. J Plant Physiol 2011, 168(18):2251-2259.

45. Preston JC, Hileman LC: SQUAMOSA-PROMOTER BINDING PROTEIN 1 initiates flowering in Antirrhinum majus through the activation of meristem identity genes. The Plant journal : for cell and molecular biology 2010, 62(4):704-712.

46. Yang W, Lou X, Li J, Pu M, Mirbahar AA, Liu D, Sun J, Zhan K, He L, Zhang A: Cloning and Functional Analysis of MADS-box Genes, TaAG-A and TaAG-B, from a Wheat K-type Cytoplasmic Male Sterile Line. Frontiers in plant science 2017, 8:1081.

47. Masiero S, Imbriano C, Ravasio F, Favaro R, Pelucchi N, Gorla MS, Mantovani R, Colombo L, Kater MM: Ternary complex formation between MADS-box transcription factors and the histone fold protein NF-YB. The Journal of biological chemistry 2002, 277(29):26429-26435.

48. Goslin K, Zheng B, Serrano-Mislata A, Rae L, Ryan PT, Kwasniewska K, Thomson B, O'Maoileidigh DS, Madueno F, Wellmer $\mathrm{F}$ et al: Transcription Factor Interplay between LEAFY and APETALA1/CAULIFLOWER during Floral Initiation. Plant physiology 2017, 174(2):1097-1109.

49. XU Lei JF-f, WANG Li-lin: Progresses on MolecularMechanisms of Flowering Transition in Arabidopsis. Acta BotBoreal 2011, 31(5):1057-1065.

50. Song SYSFZHM: Characteristics and key cultivation techniques of CCRI50 varieties China's cotton 2005, 10:25-26.

51. Tan SYSFMSZHHWR: A new variety of early early insect-resistant cotton - CCRI74. China's cotton 2010, 37(03):23.

52. Xu D: Breeding and cultivation technology of Guoxin11. China cotton society 2011:1.

53. Zhong FZH: Summary of regional trials of cotton varieties in the Yellow River basin, 1979. China's cotton 1980, 3:29-31. 
54. Gu L, Dou L, Guo Y, Wang H, Li L, Wang C, Ma L, Wei H, Yu S: The WRKY transcription factor GhWRKY27 coordinates the senescence regulatory pathway in upland cotton (Gossypium hirsutum L.). BMC plant biology 2019, 19(1):116.

55. Schmittgen TD, Livak KJ: Analyzing real-time PCR data by the comparative CT method. Nature Protocols 2008, 3(6):1101-1108.

56. Bent A: Arabidopsis thaliana Floral Dip Transformation Method. Methods in molecular biology 2006, 343:87-103.

57. Gao X, Britt RC, Jr., Shan L, He P: Agrobacterium-mediated virus-induced gene silencing assay in cotton. Journal of visualized experiments : JoVE 2011(54).

58. Gu Z, Huang C, Li F, Zhou X: A versatile system for functional analysis of genes and microRNAs in cotton. Plant biotechnology journal 2014, 12(5):638-649.

59. Sheerin DJ: Investigation of Light-Regulated Protein-Protein Interactions Using Yeast Two-Hybrid Assays. 2019, 2026:1-19.

60. Hellens RP, Allan AC, Friel EN, Bolitho K, Grafton K, Templeton MD, Karunairetnam S, Gleave AP, Laing WA: Transient expression vectors for functional genomics, quantification of promoter activity and RNA silencing in plants. Plant methods 2005, 1:13.

\section{Figures}

a

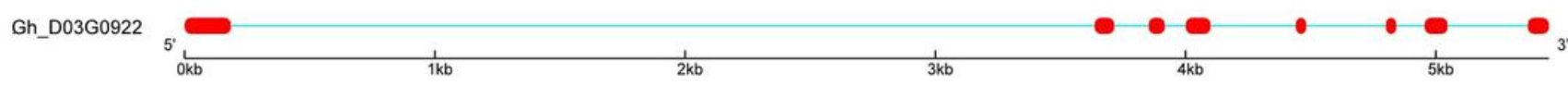

Legend:

Exon - Intron

b GhAP1

GmFULC

TaVRT-1

Consensus mgrg v lkrienkinrgvtfskrrsgllkka eisvlcda v l fs k

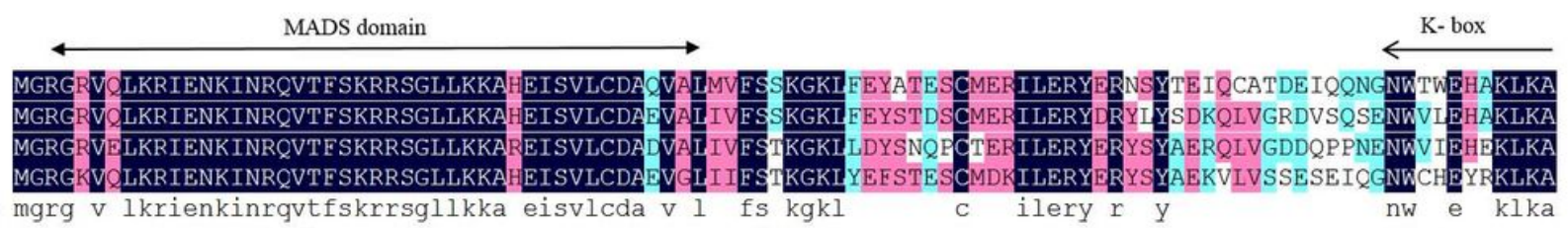

GhAP1

AtAGL8

GmFULc

TaVRT-1

Consensus
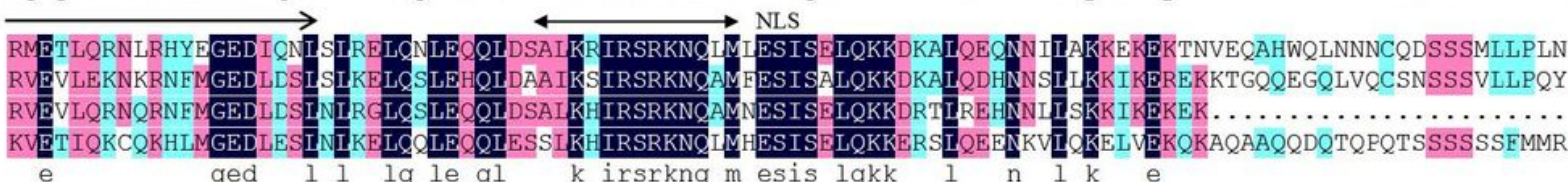

GhAP1

ISSNGREKELNETTNSGVLLPWMIRHHLE . . . . . .

CVTSSRDGFVERVGGENGGASSLTEPNSLLPAWMLRPTTTNE.

AtAGL8

DAPPAATTSIHPAASGERAEDAAVQPQAPPRTGLPLWMVSHIN

TaVRT-1

Consensus

\section{Figure 1}

Gene structure and protein sequence analysis of GhAP1. a Gene structure of GhAP1 (Gh_D03G0922). b Sequence alignment of the deduced GhAP1 protein with its homologous proteins AtAGL8 (At5g60910), GmFULc (Glyma05g07380) and TaVRT-1 (TraesCS5B02g396600). The high conserved MADS domain 
approximately 44 amino acids in length, semi-conservative $\mathrm{K}$ domain and the predicted NLS sequence were showed by the two-headed arrow. The abbreviations before the gene names of different species were as follows: At, Arabidopsis thaliana; Gh, Gossypium hirsutum; Ta, Triticum aestivum; Gm, Glycine max.

a

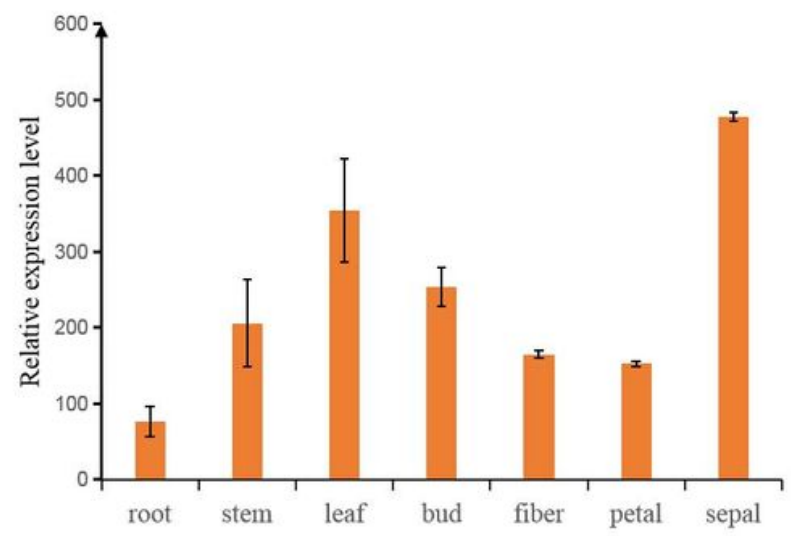

b

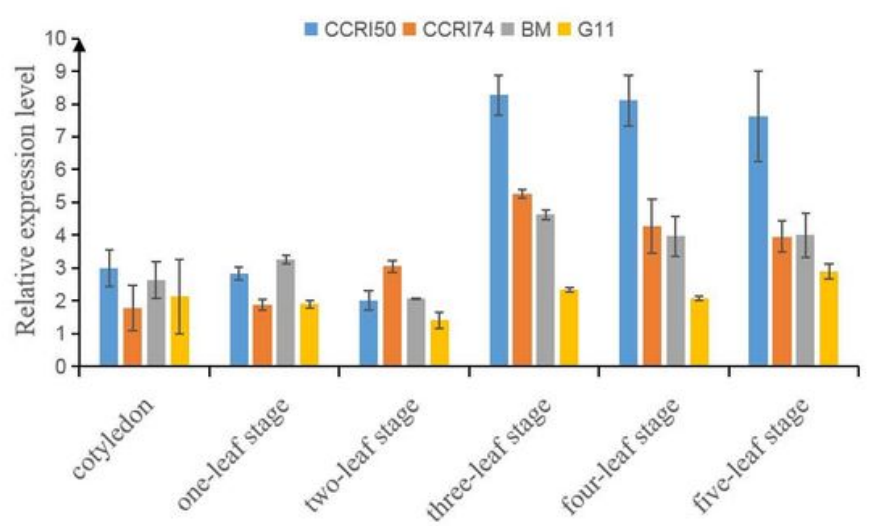

\section{Figure 2}

Expression profiles of GhAP1 in different tissues and at different stages in cotton. a Expression levels of GhAP1 in different tissues. b Expression level of GhAP1 in leaves at different developmental stages. GhActin served as the reference gene. The bars represent the means \pm standard error (SEs) from three independent experiments.

a

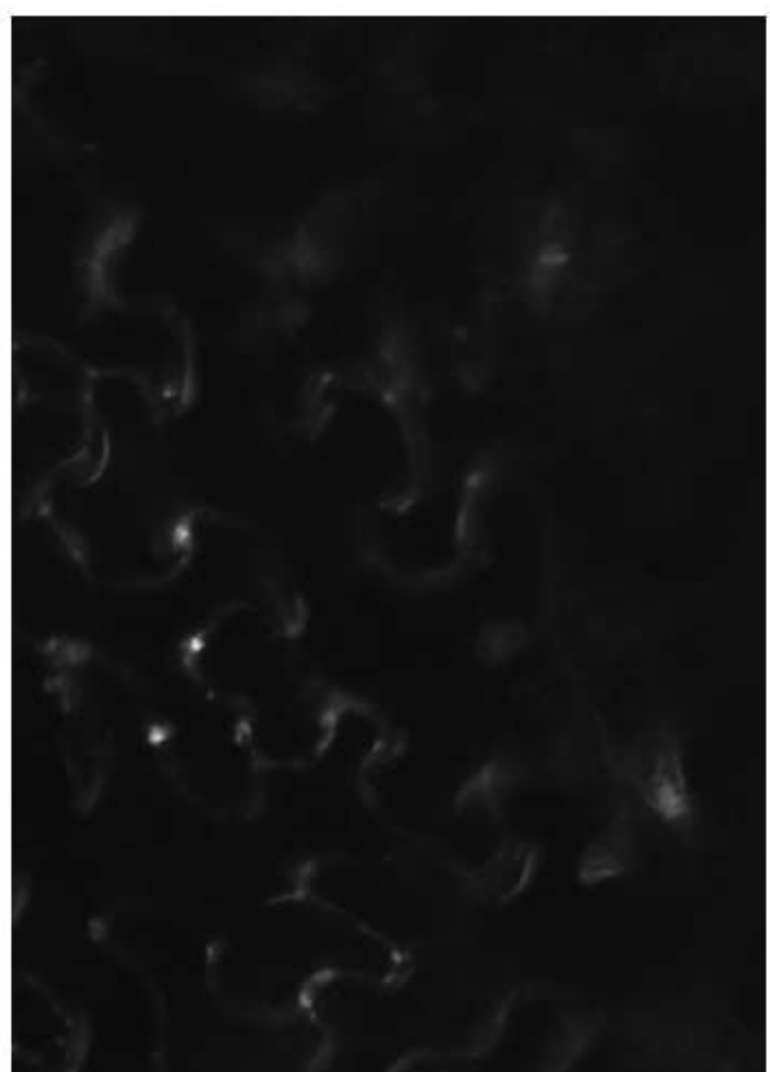

b

Figure 3 
Subcellular localization of GhAP1 protein. a Tobacco cells in bright light. b Tobacco cells with 35SGhAP1::GFP expressed instantaneously under blue excitation fluorescence.

a

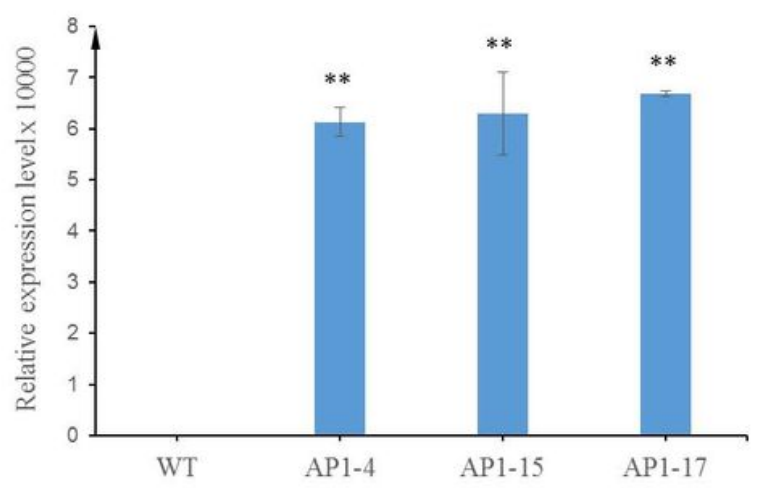

$\mathrm{b}$

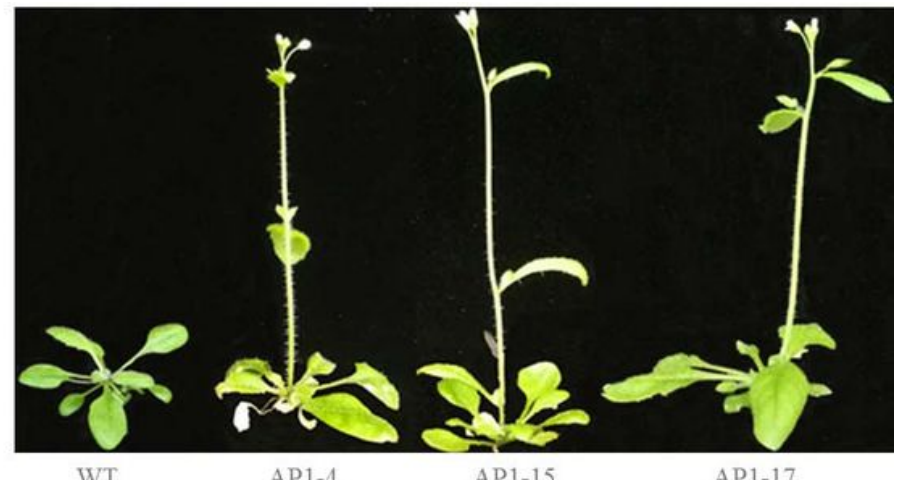

AP1-4

AP1-15

AP1-17

\section{Figure 4}

Overexpressed GhAP1 in Arabidopsis and qRT-PCR results. a qRT-PCR analysis of the transcript levels of GhAP1 in Arabidopsis. AtUBQ10 was used as a reference gene. The values represented the means \pm SDs. The significance of the data was determined using Student's t-test ( $\left.{ }^{\star} P<0.01\right)$. The experiment was conducted with three repetitions. b Early flowering phenotypes of WT plants and three transgenic lines. AP1-4, AP1-15 and AP1-17 are transgenic plants. WT is Col-0.

a

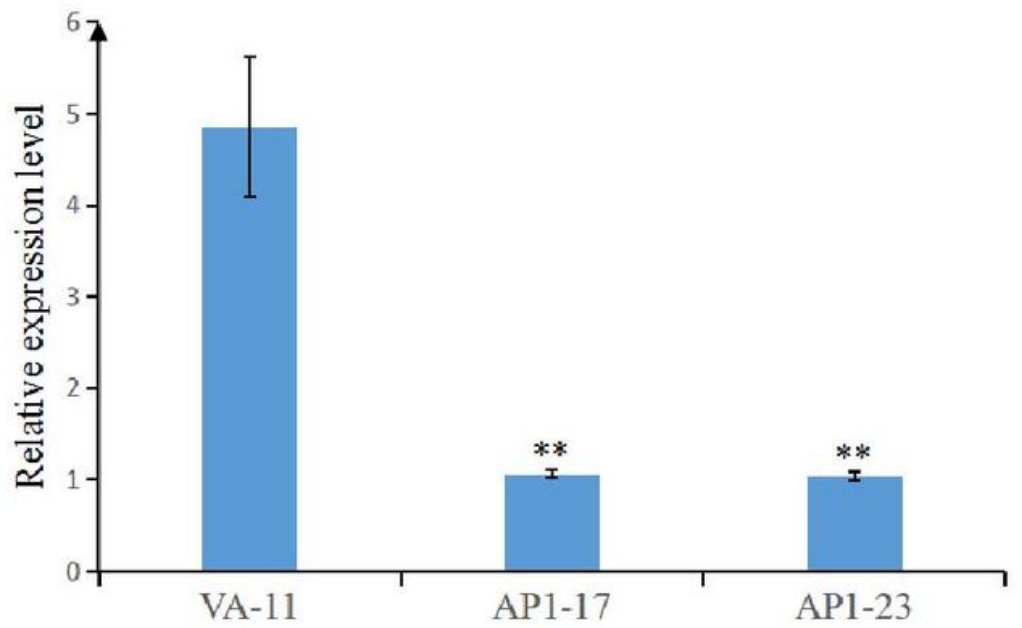

$\mathrm{b}$

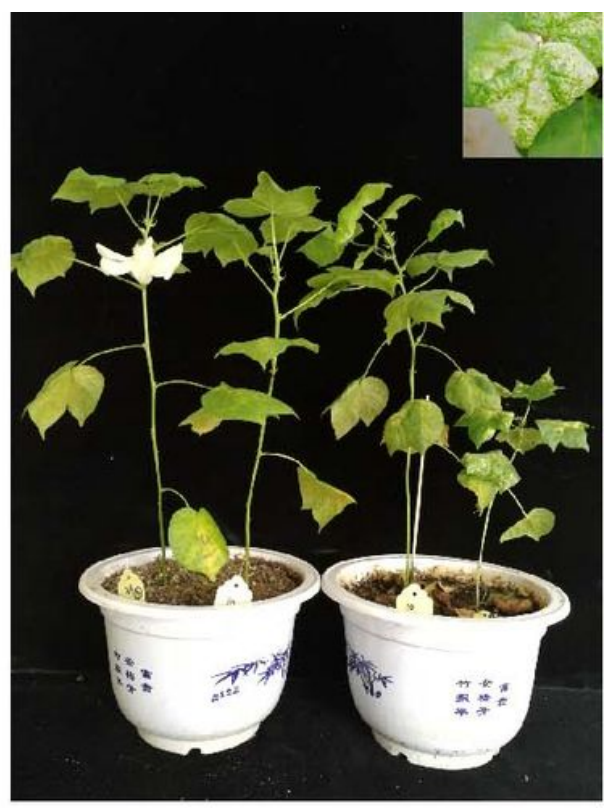

VA-11 AP1-17 AP1-23 PDS

\section{Figure 5}

VIGS phenotype and qRT-PCR analysis. a The expression levels of GhAP1 in VIGS plant and VA empty control. GhActin was served as the reference gene. The values represented the means \pm SDs from three independent experiments. Independent t-tests revealed significant $(* \star P<0.01)$ differences between the control and silenced plants. VA-11 and VA-8 were empty control; AP1-17 and AP1-23 were VIGS plants. b 
VIGS phenotype indicated positive plants flowering later than VA empty control. VA-11 was used as empty control; AP1-17 and AP1-23 were VIGS plants; PDS was pCLCrVA-PDS positive control.

a

SD-Trp

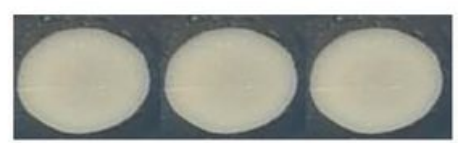

pGBKT7-GhAP1

$\mathrm{b}$

SD-Trp/-Leu

SD-Trp/-Leu/-His/-Ade/X$\alpha$-gal
SD-Trp/-His/-Ade
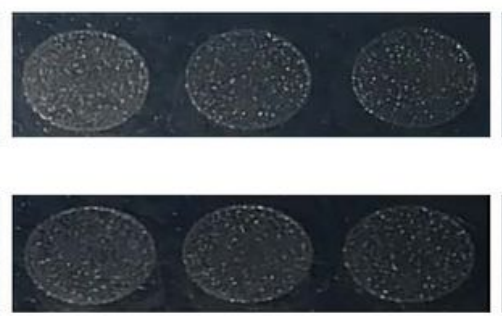

pGBKT7-laminC + pGADT7largeT
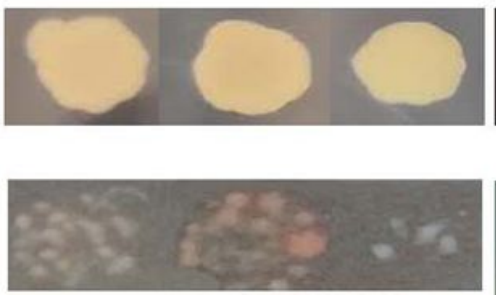

SD-Trp/-His/-Ade/X- $\alpha$-gal
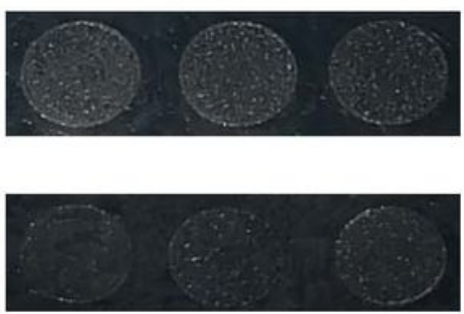

pGBKT7-GhAP1+ pGADT7-PSAT2
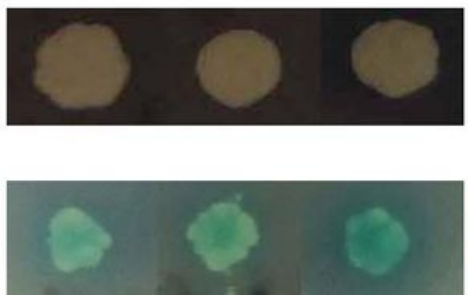

\section{Figure 6}

GhAP1 self-activation detection and GhAP1 physically interacted with GhPSAT2 in the yeast two-hybrid system. a GhAP1 self-activation detection. pGBKT7 was served as negative controls. b GhAP1 physically interacted with GhPSAT2. pGBKT7-GhAP1 and pGADT7-GhPSAT2 were co-transformed into Y2H Gold yeast cells and assayed on the SD-Trp/-Leu and SD-Trp/-His/-Ade/-Leu/X-a-gal medium . pGBKT7-p53 +pGADT7-largeT and pGBKT7-laminC + pGADT7-largeT were used as positive and negative controls, respectively. 
SD-Leu/-His

pGAD53m+p53His

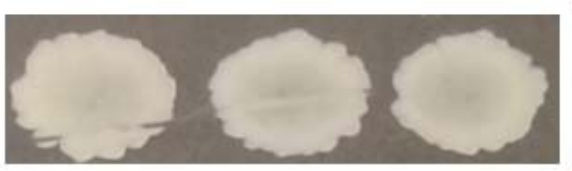

SD-Trp/-Leu

pGAD53m + pHis 2

pHis2-ProGhAP1-80bp +pGADT7-GhLFY

pHis2-ProGhAP1-1500bp + pGADT7-GhLFY

pHis2-ProGhAP1-1700bp +pGADT7-GhLFY
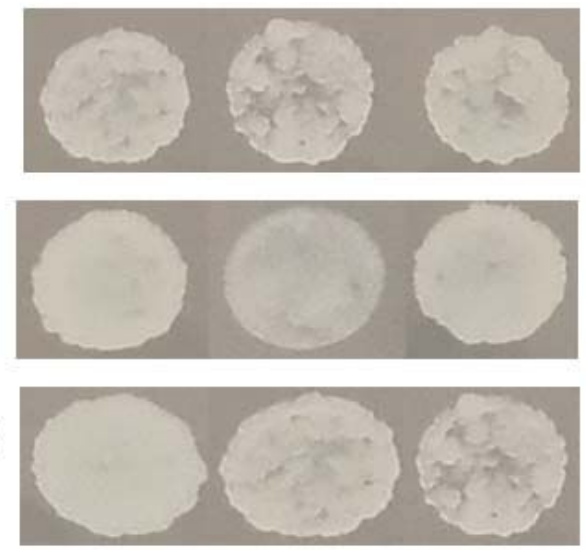

SD-Leu/-His/120mM 3-AT

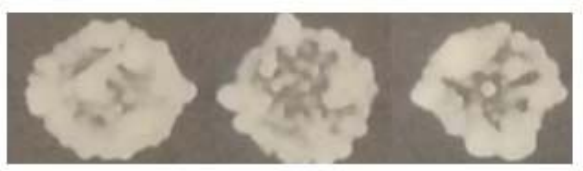

SD-Trp/-Leu/-His/120mM 3-AT
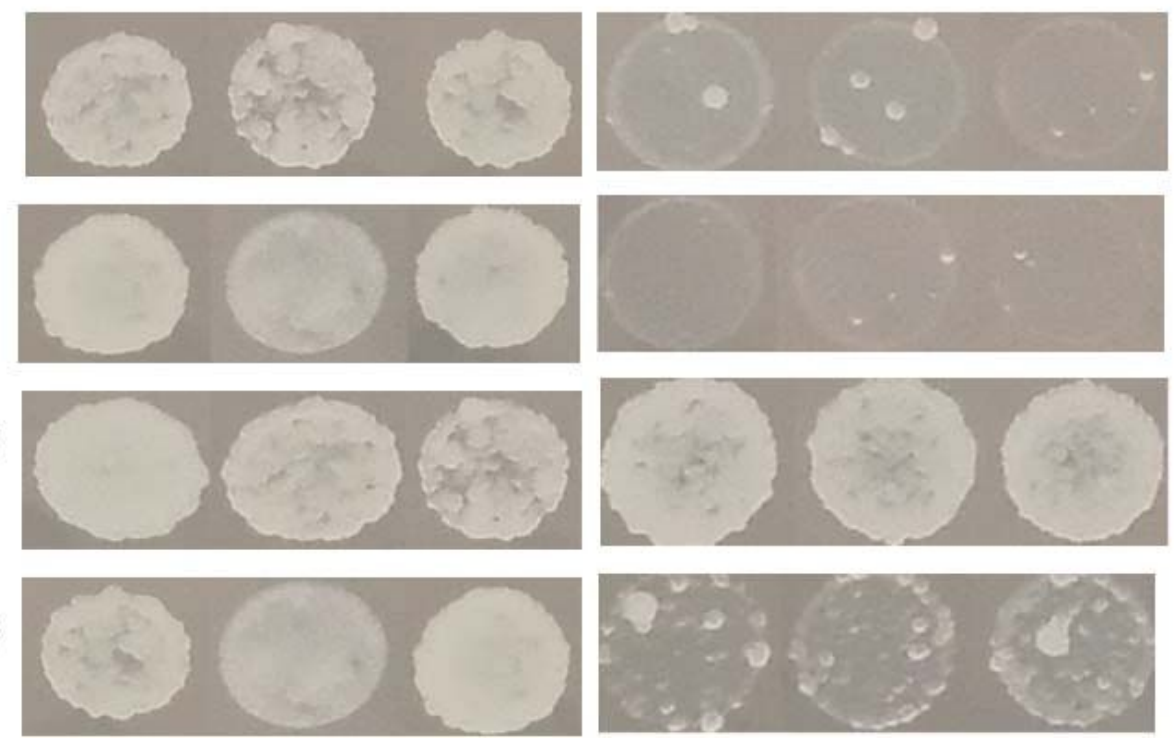

\section{Figure 7}

GhLFY bound to the promoter of GhAP1 in the yeast one-hybrid assay. pHis2-ProGhAP1 and pGADT7GhLFY were co-transformed to Y187 yeast cells. pGAD53m+p53His and pGAD53m+pHis2 were used as positive and negative controls, respectively. 
a
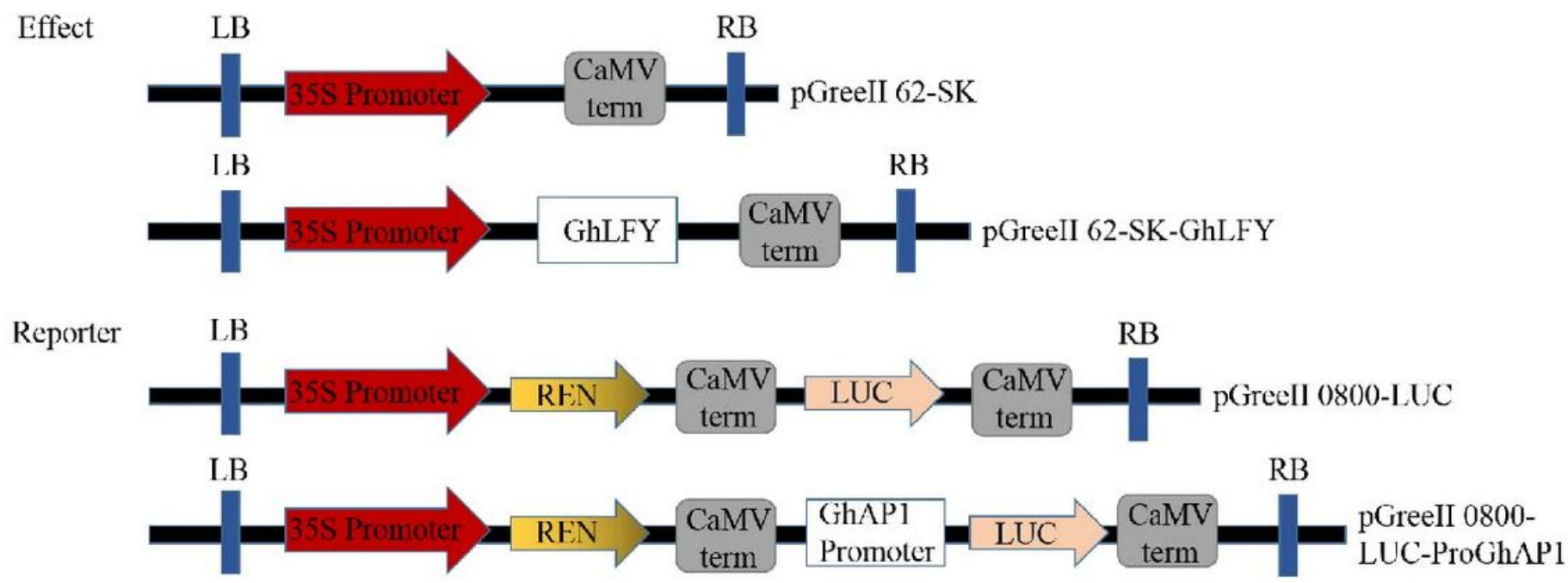

b

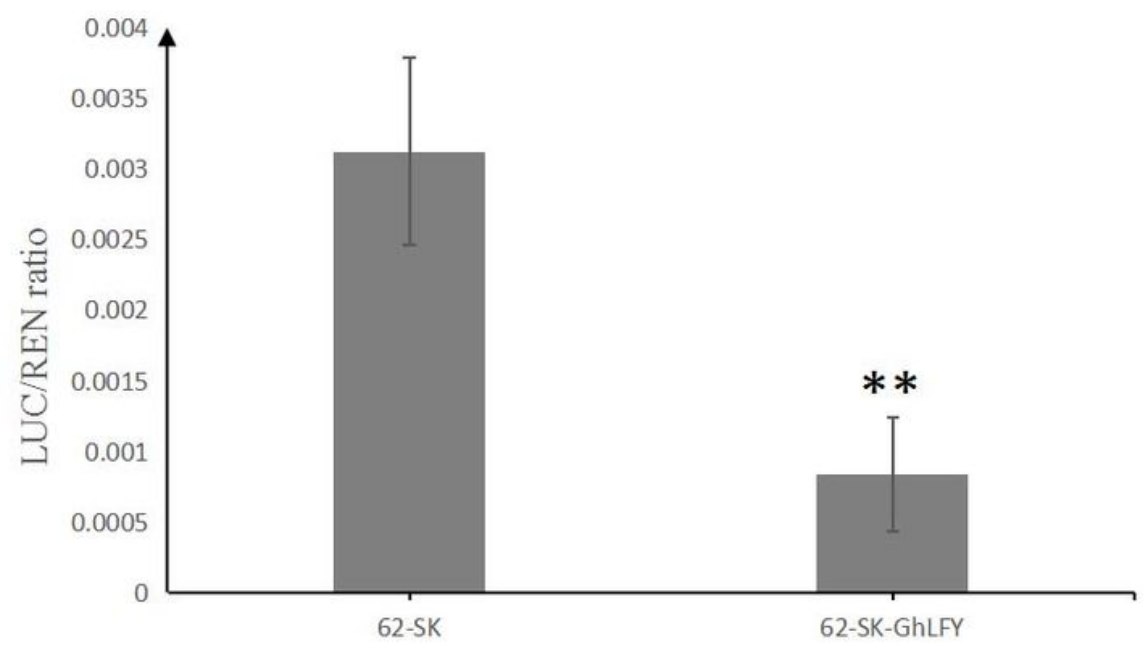

Figure 8

GhLFY inhibited the expression of GhAP1 in the dual-luciferase reporter assay. a Sketch of the effecter and reporter constructs. The GhLFY was cloned into the effecter vector pGreenll62-SK. The promoter of GhAP1 was cloned into the reporter vector pGreenII0800-LUC. b GhLFY inhibited the expression of GhAP1. The data were indicated by the ratio of LUC to REN. The values represent the means \pm SDs of six independent repeats. The significance of the data was determined using Student's t-test $(\star \star P<0.01)$.

\section{Supplementary Files}

This is a list of supplementary files associated with this preprint. Click to download.

- Additionalfile1Tables1.docx 